\title{
Three Neutrino Oscillations in Uniform Matter
}

\author{
Ara loannisian* \\ Yerevan Physics Institute, Alikhanian Br. 2, 375036 Yerevan, Armenia \\ Institute for Theoretical Physics and Modeling, 375036 Yerevan, Armenia \\ E-mail: ara.ioannisyan@cern.ch
}

\section{Stefan Pokorski}

Institute of Theoretical Physics, Faculty of Physics, University of Warsaw, ul. Pasteura 5,

PL-02-093 Warsaw, Poland

E-mail: Stefan.Pokorski@fuw.edu.pl

Following similar approaches in the past, the Schrodinger equation for three neutrino propagation in matter of constant density is solved analytically by two successive diagonalizations of $2 \times 2$ matrices. The final result for the oscillation probabilities is obtained directly in the conventional parametric form as in the vacuum but with explicit simple modification of two mixing angles $\left(\theta_{12}\right.$ and $\left.\theta_{13}\right)$ and mass eigenvalues.

ICHEP 2018, XXXIX International Conference on High Energy Physics

4-11 July 2018

COEX, Seoul, Korea

${ }^{*}$ Speaker. 
The MSW effect for the neutrino propagation in matter attracts a lot of experimental and theoretical attention.

On the theoretical side, a large number of numerical simulations of the MSW effect in matter with a constant or varying density has been performed. Although, in principle, sufficient for comparing the theory predictions with experimental data, they do not provide a transparent physical interpretation of the experimental results. Therefore, several authors have also published analytical or semi-analytical solutions to the Schroedinger equation for three neutrino propagation in matter of constant density, in various perturbative expansions [1,2,3].

We solve the Schroedinger equation in matter with constant density by two successive diagonalizations of $2 \times 2$ matrices (similar approaches have been used in the past, in particular in ref. [2] and [3]). The final result for the oscillation probabilities is obtained directly in the conventional parametric form as in the vacuum but with modified two mixing angles and mass eigenvalues ${ }^{1}$, similarly to the well known results for the two-neutrino propagation in matter. The three neutrino oscillation probabilities in matter have been presented in the same form as here in the recent ref. [4], where the earlier results obtained in ref. [3] are rewritten in this form. The form of our final results can also be obtained after some simplifications from ref.[2]. Our approach can be easily generalized to non-constant matter density by dividing the path of the neutrino trajectory in the matter to layers and assuming constant density in each layer.

In the electroweak basis the neutrino Hamiltonian is

$$
\mathscr{H}=U\left(\begin{array}{ccc}
0 & 0 & 0 \\
0 & \frac{\Delta m_{\odot}^{2}}{2 E} & 0 \\
0 & 0 & \frac{\Delta m_{a}^{2}}{2 E}
\end{array}\right) U^{\dagger}+\left(\begin{array}{ccc}
V(x) & 0 & 0 \\
0 & 0 & 0 \\
0 & 0 & 0
\end{array}\right)=U_{m}\left(\begin{array}{ccc}
0 & 0 & 0 \\
0 & \frac{\Delta m_{21}^{2}}{2 E} & 0 \\
0 & 0 & \frac{\Delta m_{31}^{2}}{2 E}
\end{array}\right) U_{m}^{\dagger}
$$

The matrix $U\left(U_{m}\right)$ is the neutrino mixing matrix in the vacuum (matter). The mass squared differences are defined as $\Delta m_{\odot}^{2} \equiv m_{2}^{2}-m_{1}^{2}\left(\approx 7.510^{-5} \mathrm{eV}^{2}\right)$ and $\Delta m_{a}^{2} \equiv m_{3}^{2}-m_{1}^{2}\left(\approx \pm 2.510^{-3} \mathrm{eV}^{2}\right.$, positive sign is for normal mass ordering and negative sign for inverted one). $\frac{\Delta m_{21}^{2}}{2 E}$ and $\frac{\Delta m_{31}^{2}}{2 E}$ are eigenvalues of the neutrino Hamiltonian (we always can add proportional to unity diagonal matrix to the neutrino Hamiltonian). $V(x)$ is the neutrino weak interaction potential energy $V=\sqrt{2} G_{F} N_{e}$ ( $N_{e}$ is electron number density) and we take it in this section to be $\mathrm{x}$-independent.

We work in the auxiliary basis $[5,6,7]$ and do two rotations for diagonalization of the neutrino Hamiltonian in eq 1.

For the mixing angles $\theta_{13}^{m}$ and $\theta_{12}^{m}$ in matter we get

$$
\sin 2 \theta_{13}^{m}=\frac{\sin 2 \theta_{13}}{\sqrt{\left(\cos 2 \theta_{13}-\varepsilon_{a}\right)^{2}+\sin ^{2} 2 \theta_{13}}}, \quad \cos 2 \theta_{13}^{m}=\frac{\cos 2 \theta_{13}-\varepsilon_{a}}{\sqrt{\left(\cos 2 \theta_{13}-\varepsilon_{a}\right)^{2}+\sin ^{2} 2 \theta_{13}}},
$$

$\sin 2 \theta_{12}^{m}=\frac{\cos \theta_{13}^{\prime} \sin 2 \theta_{12}}{\sqrt{\left(\cos 2 \theta_{12}-\varepsilon_{\odot}\right)^{2}+\cos ^{2} \theta_{13}^{\prime} \sin ^{2} 2 \theta_{12}}}, \cos 2 \theta_{12}^{m}=\frac{\cos 2 \theta_{12}-\varepsilon_{\odot}}{\sqrt{\left(\cos 2 \theta_{12}-\varepsilon_{\odot}\right)^{2}+\cos ^{2} \theta_{13}^{\prime} \sin ^{2} 2 \theta_{12}}}$

\footnotetext{
${ }^{1}$ The results of this paper have been presented as private communication by one of us (A.I) to the members of the T2HKK collaboration in December 2017.
} 
where

$$
\theta_{13}^{\prime}=\theta_{13}^{m}-\theta_{13}, \quad \varepsilon_{a}=\frac{2 E V}{\Delta m_{e e}^{2}}, \quad \varepsilon_{\odot}=\frac{2 E V}{\Delta m_{\odot}^{2}}\left(\cos ^{2} \theta_{13}^{m}+\frac{\sin ^{2} \theta_{13}^{\prime}}{\varepsilon_{a}}\right), \quad \Delta m_{e e}^{2}=\Delta m_{a}^{2}-s_{12}^{2} \Delta m_{\odot}^{2}
$$

And for differences between eigenvalues of the neutrino Hamiltonian, $\mathscr{H}$, we have

$$
\begin{aligned}
\frac{\Delta m_{21}^{2}}{2 E} & =\frac{\Delta m_{\odot}^{2}}{2 E} \sqrt{\left(\cos 2 \theta_{12}-\varepsilon_{\odot}\right)^{2}+\cos ^{2} \theta_{13}^{\prime} \sin ^{2} 2 \theta_{12}}, \\
\frac{\Delta m_{31}^{2}}{2 E} & =\frac{3}{4} \frac{\Delta m_{e e}^{2}}{2 E} \sqrt{\left(\cos 2 \theta_{13}-\varepsilon_{a}\right)^{2}+\sin ^{2} 2 \theta_{13}}+\frac{1}{4}\left[\frac{\Delta m_{e e}^{2}}{2 E}+V\right]+\frac{1}{4 E}\left(\Delta m_{21}^{2}-\Delta m_{\odot}^{2} \cos 2 \theta_{12}\right)(6)
\end{aligned}
$$

In our approximation 23 angle and the $\mathrm{CP}$ phase remain unchanged: $\theta_{23}^{m} \equiv \theta_{23}$ and $\delta^{m} \equiv \delta$ [8].

The oscillation probabilities $P_{v_{\alpha} \rightarrow v_{\beta}}(\alpha, \beta=e, \mu, \tau)$ have the same forms as for the vacuum oscillations with mass eigenstates as above and with replacements $\theta_{12} \rightarrow \theta_{12}^{m}$ and $\theta_{13} \rightarrow \theta_{13}^{m}$. For the $v_{\mu} \rightarrow v_{e}$ transition we have

$$
\begin{aligned}
P_{v_{\mu} \rightarrow v_{e}}= & \sin ^{2} 2 \theta_{13}^{m} s_{23}^{2}\left[c_{12}^{m 2} \sin ^{2} \frac{\phi_{31}}{2}+s_{12}^{m 2} \sin ^{2} \frac{\phi_{32}}{2}\right] \\
& +\frac{1}{2} c_{13}^{m} \sin 2 \theta_{13}^{m} \sin 2 \theta_{12}^{m} \sin 2 \theta_{23} \cos \delta \sin \frac{\phi_{21}}{2} \sin \frac{\phi_{31}+\phi_{32}}{2} \\
& -c_{13}^{m} \sin 2 \theta_{13}^{m} \sin 2 \theta_{12}^{m} \sin 2 \theta_{23} \sin \delta \sin \frac{\phi_{21}}{2} \sin \frac{\phi_{31}}{2} \sin \frac{\phi_{32}}{2} \\
& +\left[c_{13}^{m 2} \sin ^{2} 2 \theta_{12}^{m}\left(c_{23}^{2}-s_{23}^{2} s_{13}^{m 2}\right)+\frac{1}{4} c_{13}^{m} \sin 2 \theta_{13}^{m} \sin 4 \theta_{12}^{m} \sin 2 \theta_{23} \cos \delta\right] \sin ^{2} \frac{\phi_{21}}{2}
\end{aligned}
$$

This approximate solution is valid for all energies. For anti-neutrino oscillations $P_{\bar{v}_{\alpha} \rightarrow \bar{v}_{\beta}}, \mathrm{V} \rightarrow$ $-\mathrm{V}$ and $\delta \rightarrow-\delta$. For normal mass hierarchy $\Delta m_{a}^{2}$ is positive and for inverted mass hierarchy it is negative.

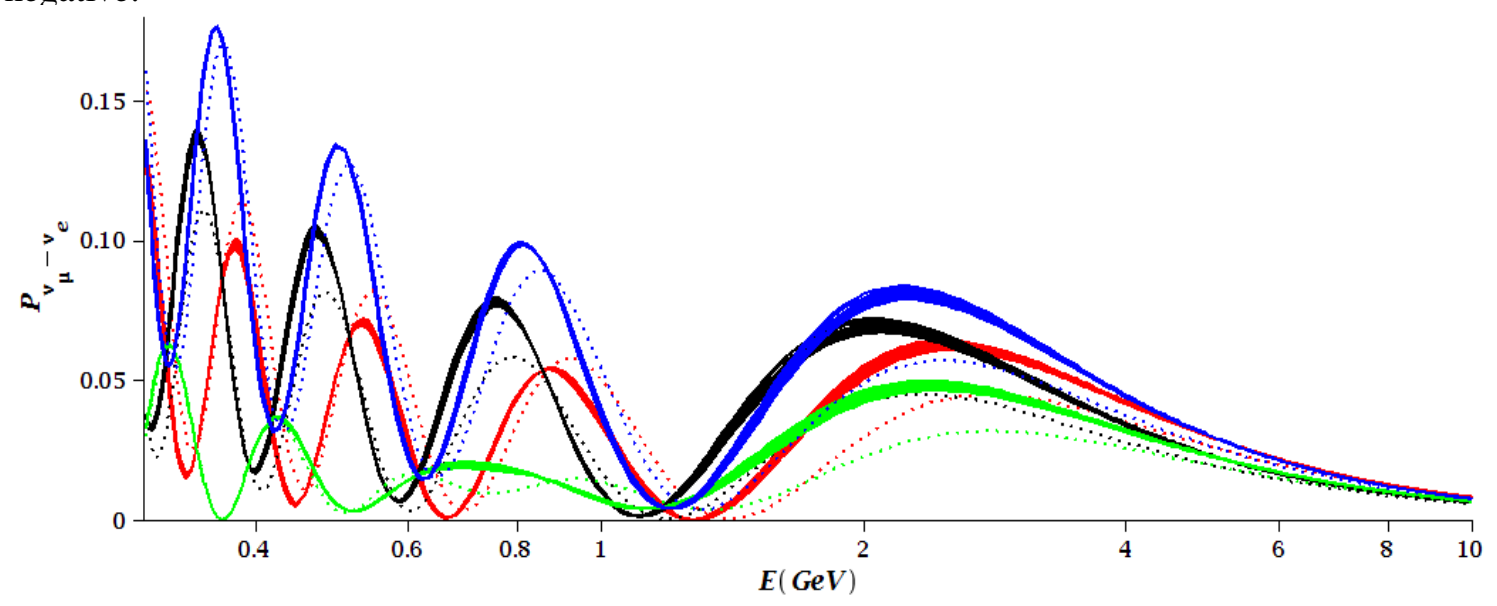

Figure 1: $v_{\mu} \rightarrow v_{e}$ oscillation probability at DUNE for normal mass hierarchy, $\delta_{c p}=0$ (red), $\delta_{c p}=\frac{\pi}{2}$ (green), $\delta_{c p}=\pi$ (black), $\delta_{c p}=-\frac{\pi}{2}$ (blue). Thickness of the plots are from varying constant/uniform matter density $2.5-3 \mathrm{~g} / \mathrm{cm}^{3}$. Dotted plots are for vacuum oscillations

Our solutions are illustrated in Fig. 1 for $v_{\mu} \rightarrow v_{e}$ oscillation at DUNE distance for several values of $\delta_{C P}$ and compared with the oscillation probabilities in the vacuum, shown by the dotted curves. 
The most important effect is the dependence of the oscillation probability on the angle $\theta_{13}$ which has larger (smaller) values in matter than in the vacuum for normal (inverted) neutrino mass hierarchies (and opposite for antineutrinos). Thus the oscillation probabilities have larger(lower) oscillation amplitudes for normal (inverted) neutrino mass hierarchies (and opposite for antineutrinos). In oder words the matter of the Earth is amplifying the effect of the mass ordering on neutrino oscillations. The dependence on the angle $\theta_{13}$ enters multiplicatively in the first three terms of eq (7), whereas the fourth term is small in the region of the first maximum. Therefore the matter effects relative to the oscillations in the vacuum do not depend on the value of $\delta_{C P}$, as it is seen in Fig. 1 . Moving to the next resonances (lower energies) the difference between oscillations in matter and in the vaccuum remain qualitatively similar, although some small differences can be seen due to the fact that the change in the angle $\theta_{13}$ is smaller.

In Fig. 2 we show the accuracy of the analytical solutions comparing them with numeri$\mathrm{cal} / \mathrm{exact}$ results.

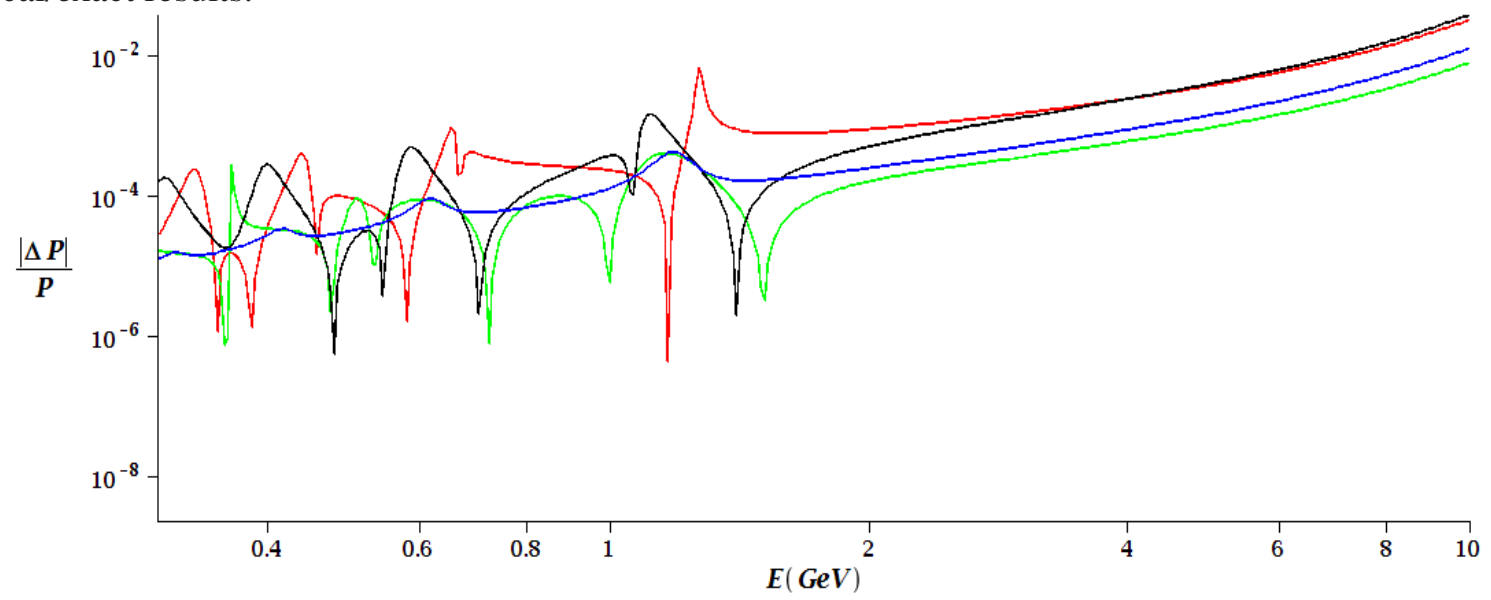

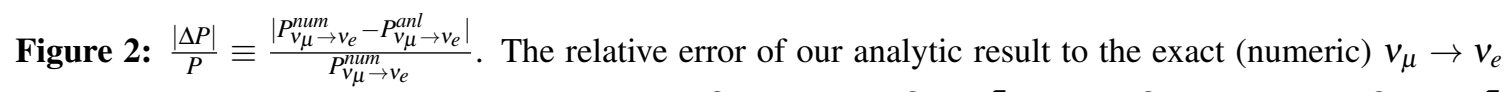
oscillation probability for normal mass hierarchy, $\delta_{c p}=0$ (red), $\delta_{c p}=\frac{\pi}{2}$ (green), $\delta_{c p}=\pi$ (black), $\delta_{c p}=-\frac{\pi}{2}$ (blue). Matter density $2.6 \mathrm{~g} / \mathrm{cm}^{3}$.

\section{References}

[1] S. T. Petcov, Phys. Lett. B 214 (1988) 259. E. K. Akhmedov, R. Johansson, M. Lindner, T. Ohlsson and T. Schwetz, JHEP 0404 (2004) 078. A. Cervera, A. Donini, M. B. Gavela, J. J. Gomez Cadenas, P. Hernandez, O. Mena and S. Rigolin, Nucl. Phys. B 579 (2000) 17 Erratum: [Nucl. Phys. B 593 (2001) 731]. H. Nunokawa, S. J. Parke and J. W. F. Valle, Prog. Part. Nucl. Phys. 60 (2008) 338

[2] M. Blennow and A. Y. Smirnov, Adv. High Energy Phys. 2013 (2013) 972485

[3] P. B. Denton, H. Minakata and S. J. Parke, JHEP 1606 (2016) 051

[4] P. B. Denton, H. Minakata and S. J. Parke, arXiv:1801.06514 [hep-ph].

[5] P. I. Krastev and S. T. Petcov, Phys. Lett. B 205 (1988) 84.

[6] O. L. G. Peres and A. Y. Smirnov, Nucl. Phys. B 680 (2004) 479

[7] A. Ioannisian and S. Pokorski, Phys. Lett. B 782 (2018) 641

[8] S. T. Petcov and Y. L. Zhou, Phys. Lett. B 785 (2018) 95 